\title{
FORMACIÓN CIUDADANA Y CONSTRUCCIÓN DE PAZ CON EQUIDAD. RETOS ACTUALES DE LA EDUCACIÓN MEDIA SUPERIOR
}

\author{
EDUCATING CITIZENRY AND \\ PEACE BUILDING WITH EQUITY. CURRENT \\ CHALLENGES OF HIGH \\ SCHOOL EDUCATION
}

Xicoténcatl Martínez Ruiz Sylvia van Dijk

Doctor en Filosofía, Lancaster University, Inglaterra. Investigador del Instituto Nacional de Ciencias Penales, Dirección de Investigación, México. Miembro del Sistema Nacional de Investigadores, nivel 1.

xicotencatlm@gmail.com

Doctora en Ciencias de la Educación. Profesora de tiempo completo en el Departamento de Educación de la Universidad de Guanajuato. Miembro del Sistema Nacional de Investigadores.

dijkhoog@prodigy.net.mx

\section{RESUMEN}

A la luz del imperativo de la Educación Media Superior de México (EMS) que señala, como una de sus cuatro funciones, la educación ciudadana, se brinda un marco de discusión teórica sobre ciudadanía. Se cuestiona el desarrollo depredador y generador de inequidades a 
todos los niveles. Se vincula el ejercicio ciudadano con la discusión actual entre bienestar y desarrollo. Asimismo, se retoma el marco que establece la Convención Internacional de los Derechos del Niño (ONU, 1989) para contextualizar la EMS en México, esbozando problemáticas en torno a la formación ciudadana que se visualizan desde la organización actual y desde el marco curricular común planteado para este nivel. Se retoma la manera en que se conforman los programas y se contrastan con los propósitos generales de este nivel, planteados por la Secretaría de Educación Pública. Enseguida, desde una perspectiva "glocal», se discute como podría ser la formación ciudadana de las siguientes generaciones en la EMS. La primera parte del trabajo concluye con un planteamiento para incorporar la formación ciudadana a la EMS. La segunda, analiza la idea de ciudadanía desde la construcción de paz con equidad y ética, desde una cuestión clave en nuestro siglo: ¿qué implicaciones tienen las actuales revoluciones tecnológicas en la Educación Media Superior y la formación ciudadana? Se esbozan tres sugerencias para la revisión de un marco curricular común en la EMS: a) la no violencia para construir una cultura y educación de paz, aplicada al entorno social; b) equidad en la educación y, c) Ética Digital e integridad académico-laboral.

Palabras clave: ciudadanía, ciudadanía infantil, Educación Media Superior, cultura de paz, educación para la paz.

\section{ABSTRACT}

The Mexican school system has defined as one of the four functions for the last three years of compulsory education (15-18 years) citizenship education. From this imperative, we discuss theoretical notions of citizenship. Depredating development that creates inequalities at all levels is questioned. Citizenship is discussed within the framework of the relationship between well-being and development. The International Convention of the Rights of the Child (UN, 1989) is taken up in the context of the actual organization of educational institutions and prescribed curriculum, pointing out obstacles to make children's rights a daily practice. 
The proposed new programs are analyzed contrasting them with the general goals and functions as stated by the educational authority. We discuss possibilities for citizenship education for the next generations in schools from a global perspective and conclude this first part with a proposal for citizenship education.

In the second part we take up the need to see citizenship education as a means to build up peace with equity and ethics, from a key perspective in our times ¿what consequences do the ongoing technological revolutions in our times have for the last years of high school and citizenship education? A quick presentation is done of three elements that could enrich the actual programs for high school: a) nonviolence to build up a culture and education for peace applied to the social context of schools; b) equity in education and, c) digital ethics and academic-professional integrity.

Keywords: citizenship, children's citizenship, high-school education, cultural peace, peace education.

\section{INTRODUCCIÓN}

La última reforma educativa, que entró en vigor en 2018 (SEP, 2017, p. 12), plantea que la formación ciudadana es una de las cuatro funciones de la EMS. Resulta pertinente, entonces, discutir qué entendemos por ciudadanía y, en específico, por ciudadanía infantil que incluye a los jóvenes entre 15 y 18 años. Enseguida se ofrece un breve análisis del plan de estudios presentado por la SEP para la EMS, y se cierra la reflexión con propuestas de educación para la paz, Ética y equidad a manera de conclusión.

\section{CIUDADANÍA Y ENTORNOS ACTUALES}

En Occidente, la ciudadanía tiene su origen en Grecia y su esencia es mantener un orden social para el bien común; esta es la responsabilidad de todo ciudadano, de acuerdo con su profesión o lugar en la 
sociedad. Sin embargo, se asume también que es su derecho ya que le otorga felicidad al vivir en comunidad (Aristóteles, 2000). En el transcurso de la Historia, la búsqueda de justicia entre diferentes grupos sociales ha reclamado derechos ciudadanos para todos y, a partir de la Revolución Francesa, la igualdad de todas las personas que conforman una sociedad. Cada época imagina formas diferentes de ejercer la ciudadanía; todas consideran la participación del individuo en búsqueda del bien común, aunque este último se entienda desde la perspectiva de los grupos en el poder. El ejercicio de la ciudadanía requiere de cada individuo un esfuerzo, compromiso y sentido de responsabilidad —que presupone capacidades personales como la escucha, argumentación, empatía, reflexión, autorregulación, metacognición-para conducir un diálogo que promueva el consenso y la toma de decisiones colegiadas. El ejercicio ciudadano supone, asimismo, una autoridad que posee las mismas capacidades y que se abstiene del uso arbitrario del poder, así como de la opresión o represión de personas y grupos minoritarios u opositores.

En el siglo XX la ciudadanía se vinculó al ejercicio de los derechos humanos y esta relación estrecha entre ambos conceptos también contribuyó al énfasis en el individuo, que junto con la fragmentación del conocimiento y de los grupos sociales, ha desdibujado la búsqueda del bien común. La época postcolonial y la búsqueda de justicia en diferentes naciones, así como las dinámicas migratorias de la segunda mitad del siglo XX, dejaron en claro que, a pesar de las tendencias uniformizantes de la globalización, los universalismos deben repensarse, pues los grupos minoritarios de las sociedades tienden a ser excluidos y marginados, por lo que no gozan de los derechos humanos ni del ejercicio ciudadano (de Sousa Santos, 1997). El afán universalizante ha hecho invisible otras perspectivas de ciudadanía más centradas en el bien común que en el individuo, como es el caso de las culturas originarias.

El ejercicio de la libertad es otro de esos ideales que desde la tradición liberal en Occidente se introdujo como un máximo bien a aspirar. Incluso desde la teoría económica, Amartya Sen (1982) plantea el desarrollo de las capacidades humanas al máximo potencial, con la finalidad de que los individuos elijan con la mayor libertad posible su modo de vida, sin dañar al otro. Esta acepción de libertad —en torno 
a las oportunidades de desarrollo personal en los ámbitos socioeconómico, cultural y político- nuevamente centra toda la atención en el individuo. Las circunstancias del entorno están en función de la persona, asumiendo que esto la llevará a alcanzar bienestar y a sentir felicidad y paz.

Como bien plantean Kincheloe y Steinberg (2012): «El reto del nuevo siglo XXI sigue siendo el de definir los derechos humanos en términos capaces de sostener el principio de la igualdad a partir del reconocimiento de la diversidad» (p. 12).

El concepto de ciudadanía se ha desgastado, aunque en todas las naciones existen grupos sociales cuya participación activa busca equilibrios, justicia, y distribución de bienes y servicios, considerados bienes colectivos a alcanzar. La ironía es que, mientras más se lucha por el acceso equitativo a servicios públicos, los gobiernos de todo el mundo están privatizándolos, creando de esta manera mayores inequidades. En proporción, los ciudadanos con pocos medios económicos pagan un mayor porcentaje de sus ingresos por el acceso a servicios básicos como agua potable, comunicación, educación, salud, conectividad, entre otros.

El ejercicio democrático de la ciudadanía no se consigue por un acto de buena voluntad ni por decreto; requiere de un esfuerzo sistemático en el que se ejerciten las capacidades arriba mencionadas. A medida que nuestras sociedades se complejizan, el ejercicio de la participación ciudadana es más demandante. La capacidad de pensarse uno mismo y de pensar las colectividades en las que vivimos, desentrañando la calidad de las relaciones así como los mecanismos de exclusión, y la complejidad de los grupos que las conforman, es un reto cotidiano. La dialogicidad es múltiple y surge en contextos donde se juegan poderes, a veces difícilmente identificables. En estos esfuerzos por aprehender sociedades diversas, pero interconectadas entre sí, se introdujo el término de ciudadanía «glocal» (Pries, 2005) que exige una atención consciente en dos vertientes: las acciones locales dentro de un territorio que impactan en nuestro entorno inmediato y su repercusión a nivel planetario. Esta doble dimensión interactuante es fundamental para comprender la economía, cultura, ecología y política en cualquier lugar 
del mundo. Las realidades locales y globales se ven atravesadas, a su vez, por redes, mayoritariamente virtuales, que son «espacios fluidos» con gran impacto en nuestra percepción de la realidad.

Como fue retomado por Martínez Ruiz (2017) del Onlife Manifesto (2015), la tecnología modifica nuestra percepción de la realidad y, con ello, nuestras formas de vida, a las que también es necesario proporcionar una respuesta ciudadana. Los avances en materia de comunicación y robotización, así como de flujos de información, desdibujan fronteras: ya no se distingue lo real de lo virtual, así como se disuelven diferencias entre lo humano, la máquina con inteligencia artificial y la naturaleza. Por otro lado, la humanidad ha transitado de una falta de información a una saturación informática y la generación de la riqueza se desplaza de los objetos a las interacciones.

¿Cómo reaccionan las sociedades frente a estos cambios tan profundos, cuyos impactos apenas se vislumbran? Ciertamente, solo las élites internacionalmente conectadas son las que deciden en estos ámbitos y las mayorías siguen excluidas de ejercer influencia alguna en las nuevas configuraciones de sus entornos. Frente a los embates de estas realidades emergentes sobre los diferentes grupos sociales, las personas sufren o disfrutan, se adaptan y encuentran formas de sobrevivencia. Los gobiernos de las naciones tampoco han tenido gran injerencia, pues simplemente observan lo que les representa un crecimiento en el Producto Interno Bruto (PIB) o padecen las mermas que estos nuevos desarrollos tienen sobre formas de producción, comercialización, oferta de bienes y servicios. La velocidad de la innovación tecnológica, desarrollada bajo su propia inercia, rebasa la capacidad de las sociedades de pensarlas y de reflexionar sobre sus impactos en el bienestar, en la salud, en el medio ambiente y en las economías.

Los retos para las ciudadanías son inmensos y requieren de capacidades poco cultivadas en los últimos años, como es el autoconocimiento, o como afirma Morin (1999), conocer y pensar nuestra condición humana, repensar los paradigmas de las ciencias, desarrollar la contemplación, la observación, la escucha, la empatía, la solidaridad con la vida, la compasión, la imaginación-creativa, la interiorización y los procesos de atención consciente (Martínez Ruiz, 2016). Todos los habitantes del 
planeta requerimos ampliar nuestra resiliencia y adaptabilidad, pero más que esto la capacidad de dialogar con múltiples actores que poseen diferentes marcos de referencia -incluyendo a nuestra Madre Tierra-, para pensar y enfrentar con amor la vida. El diálogo con la Madre Tierra implica, como bien señala Leonardo Boff (2012), «amarla, respetarla y reverenciarla» (s/p); no se le puede vender, mercantilizar, explotar económicamente o instrumentarla; es conciencia inteligente con la que hay que dialogar.

En resumen, para ejercer una ciudadanía a la altura de los retos que enfrentamos como humanidad, es necesario acrecentar competencias del pensamiento crítico, imaginativo y creativo, realizando cambios paradigmáticos para dialogar con sujetos nuevos (como son las personas menores de 18 años) o la Madre Tierra que ha sido explotada, vendida, apropiada, acaparada, sin respetar su dinamismo, sus lenguajes, su vida, sus manifestaciones creativas.

Dada la enorme diversidad de circunstancias externas e internas, los grupos sociales deben hallar formas propias y creativas de ejercer su ciudadanía, de participar activamente en sus entornos inmediatos, siempre con la mira en el planeta completo. La humanidad entera debe reconstruir sus entornos descompuestos por violencia, depredación y allanamiento de grupos específicos.

Fenómenos como la enorme movilización de niños y adolescentes entre 10 y 18 años, que ocuparon las calles de grandes ciudades en Estados Unidos de Norteamérica, manifestó un grito desesperado que implicó un gran esfuerzo en logística y en recursos. Sin embargo, el gobierno de ese país ha hecho oídos sordos a su petición de cambiar las leyes de armas (Pardo, 2018).

El ejercicio ciudadano se vincula al bienestar y la felicidad de quienes conforman las sociedades. Entendemos por «bienestar» las condiciones de vida que cubren las necesidades básicas de sobrevivencia, identidad y cultura: alimentación, vestido, vivienda, cuidados de salud, educación, movilidad, comunicación, facilidades adecuadas de esparcimiento en áreas verdes y en manifestaciones culturales y artísticas. La felicidad es más subjetiva, pero entre sus cualidades más 
importantes están: sentido de vida, confianza en los núcleos familiares y en las instituciones sociales.

Actualmente, las sociedades experimentan grandes disrupciones y fracturas, desde el núcleo de las familias hasta la percepción política y económica de los ciudadanos. Las democracias representativas son modelos rebasados; los gobiernos no logran ganarse la confianza de sus ciudadanos y tampoco muestran capacidad de apertura de espacios reales de participación para los diversos grupos poblacionales. Los jóvenes sienten esto más que cualquier otro grupo, pues viven la contradicción entre el discurso oficial de oportunidades educativas para todos y los mecanismos de expulsión y exclusión en las escuelas, a la vez que cuentan con pocos o nulos espacios reales de participación y toma de decisiones.

En los últimos años, los jóvenes han descubierto un nuevo espacio para la participación, en ocasiones de manera adictiva: las redes sociales donde viven la libertad de expresión, encuentran pares que los escuchan, asumen identidades ficticias, juegan videojuegos, «matan» el tiempo para bajar niveles de ansiedad y ocio, entran en contacto con el mundo... Las tecnologías de información y comunicación proporcionan acceso a información antaño inasequible para grandes grupos sociales $\mathrm{y}$, en muchos sentidos, esta realidad emergente sacude diariamente las realidades educativas.

Las instituciones educativas siguen siendo espacios cerrados, desvinculados - en multitud de sentidos - de la realidad en la que se hallan inmersas; a ellas asisten ciudadanos menores de dieciocho años y, por ello, es indispensable realizar también una breve reflexión en torno a la noción de ciudadanía infantil.

\section{Ciudadanía infantil}

Desde 1990, los niños de cero a dieciocho años son ciudadanos, sujetos de derechos con prerrogativas especiales. La Convención Internacional de los Derechos del Niño (ONU, 1989) refleja el logro consensuado después de diez años de discusión internacional. Es un documento 
único en su categoría de derechos humanos, ya que conjuga los derechos individuales, socioculturales, políticos y económicos de las nuevas generaciones. En México, este documento es parte del marco legal vigente de máxima jerarquía, junto con la Constitución Mexicana.

La Convención responde al paradigma de la complejidad y no puede leerse de forma lineal ni fragmentada. Cada artículo ha de verse como una cara que ilumina todas las demás. Así, por ejemplo, cuando se habla de identidad, esta debe tomarse en cuenta, como lo marca el documento, en todos los demás artículos enunciados. El conjunto de artículos puede visualizarse como una esfera fractal. Esta atención en lo particular y en lo general es, al mismo tiempo, reto para nuestras mentes que han sido entrenadas en el análisis y en la fragmentación. La síntesis - la mirada holista- resulta difícil dada la formación de los profesionales. Así, por ejemplo, el maestro se deslinda de la participación del estudiante o de la educación en el cuidado de su salud, o de la protección contra la violencia. Esta complejidad se multiplica si, además, el ejercicio de los derechos no se concreta de la misma manera para un niño de dos años que para uno de cuatro, seis, o dieciséis, dado el acelerado crecimiento y la enorme cantidad de capacidades que adquiere el ser humano durante los primeros años de vida. La Convención se asume de acuerdo con la evolución de las capacidades del niño. Si queremos visualizar una imagen que refleje el dinamismo inherente en este documento legal, pensemos que la esfera arriba mencionada se transforma en una espiral en constante movimiento.

La no discriminación (ONU, 1989, art. 2), el interés superior del niño (ONU, 1989, art. 3), su participación genuina —incluyendo libertad de pensamiento, conciencia y religión-, así como de asociación y reunión pacífica (ONU, 1989, arts. 12-15) y el derecho al desarrollo al máximo de sus potencialidades (ONU, 1989, art. 6) son artículos que aplican a todos los demás y se implican entre sí. El conjunto de artículos provee al niño de todo aquello que asegura una vida digna, lo protege contra toda intromisión en su privacidad y de violencia de cualquier tipo, y le asegura la participación en la vida pública local y planetaria. Los adultos son garantes de estos derechos y el Estado es el último responsable de asegurar su cumplimiento al buscar generar los mecanismos requeridos para que los padres de familia provean, en condiciones dignas, la 
supervivencia de sus hijos y para asegurar el acceso a servicios como salud, educación, protección legal, entre otros. En varios sentidos, la Convención representa una ruptura paradigmática con la tradición, tanto en Occidente como en las demás regiones y culturas del planeta:

a) Si se concibe al niño como sujeto de derechos se le libera de esta visión caritativa de un sujeto con necesidades; el cumplimiento de sus derechos ya no está en manos de la buena voluntad de los adultos, sino que el Estado se obliga a entrar en la lógica de establecer políticas públicas y mecanismos reglamentarios que hagan imperativos y exigibles la provisión, protección y los espacios de participación establecidos en el documento legal (Abramovich, 2004).

b) Con este marco legal, quienes atienden a personas menores de 18 años son garantes de sus derechos: servidores de salud, de recreación, de cultura, de educación. Es decir, entre otros servidores de la niñez, los maestros son garantes de estos derechos y muy pocos lo saben, no conocen a fondo las implicaciones del documento y no asumen su responsabilidad.

c) La noción de niñez ha variado enormemente en lo que conocemos como Historia: desde las prácticas socialmente aceptadas del infanticidio, la explotación laboral y la violencia en los procesos de desarrollo (Ariès, 1987). La humanidad ha reconocido, finalmente, que cada niño es un ser único, valioso y digno de cuidados y oportunidades de desarrollo. Pasa de ser objeto de compasión-represión a ser sujeto de derechos, como un ser humano completo al que no le falta algo para tener opinión, sentimientos, valores a partir de los cuales construye su propio desarrollo (García Méndez, 1998). Se reconoce su vulnerabilidad frente al adulto y, por ello, debe protegérsele de tantos atropellos a los que ha estado sometido; sin embargo, se reconoce su vitalidad, fuerza, resiliencia y poder de transformar las sociedades en las que participa. La propia noción de niñez recoge este dinamismo que se desencadena a partir de la interacción constante de fuerza y vulnerabilidad.

Este viraje en la concepción de niñez fue producto de largas discusiones entre especialistas de diversas disciplinas, pertenecientes a todas 
las regiones y culturas del mundo. Es un documento que equilibra los bienes individuales y socioculturales, pero también abre la puerta a una participación genuina y responsable. Los niños son igualmente responsables de conducirse sin pisar los derechos de sus prójimos, sean estos adultos o niños; en términos legales, también son garantes de los derechos que ellos mismos gozan, con excepción de los derechos de provisión ya que ellos, por su condición de desarrollo, no pueden proveer a los demás. En otras palabras, no estamos frente a la dialéctica dicotómica tradicional en términos jurídicos de derechos y obligaciones, sino que se parte del paradigma complejo en el que múltiples relaciones determinan la existencia de la persona, en donde muchas relaciones son recíprocas, pero otras transitan en planos más complejos de Ética, Moral, responsabilidades diferenciadas, capacidades diferentes, por mencionar algunos. Ciertamente, el principio de respeto y amor a las nuevas generaciones, incluye la contención que los adultos deben brindar a los niños. Como han señalado otros estudiosos del tema, el campo de la infancia se mueve entre la reproducción social, la resistencia y la transformación (Marre, 2014).

La ciudadanía infantil es un concepto nuevo (Invernizzi y Milne, 2005) cuyas implicaciones y ejercicio se han explorado poco, en virtud de que los adultos no han abierto estos espacios y las autoridades de las naciones tampoco. Los niños pocas veces son invitados a participar en la planeación urbana, en el desarrollo curricular, en el diseño de parques. Aún son escasas las instituciones que toman en cuenta sus necesidades en la disposición y el tamaño de lavamanos, retretes, apagadores, entre otros. Es una agenda pendiente, pero para efectos de la nueva reforma educativa, asume muchas consecuencias. Formar para la ciudadanía requiere del ejercicio ciudadano de los niños, y de la exploración de sus alcances y limitaciones para cada grupo etario, y para los diferentes contextos en los que crecen y se desarrollan.

La ciudadanía infantil supone un compromiso personal de involucrarse en la comunidad, de buscar espacios para contribuir al bien común, de participar activamente en todos los ámbitos de su vida con responsabilidad, pero también con arrojo y deseos de transformación en su persona, familia, escuela, colonia/barrio/comunidad, entorno urbano o rural, medio ambiente, manifestaciones artísticas, socioculturales, medios de comunicación masiva (radio, TV, redes sociales...); 
en fin, integrarse activamente a la sociedad a la que pertenece. Cada niño representa el reto de facilitar la construcción de un ser complejo que participa activamente en su desarrollo y creación. Esto no sucede si los adultos no les abren espacios y no se comprometen a entablar un diálogo real con las generaciones jóvenes. Mientras se siga esperando adaptación y obediencia por parte de niños y adolescentes, no se abrirán espacios para el ejercicio de la ciudadanía de las nuevas generaciones. Como se afirmó al inicio, esto requiere de muchas competencias complejas a acrecentar en nuestro país, tanto por parte de los adultos, como por parte de los seres humanos en pleno y acelerado desenvolvimiento. A la luz de estas perspectivas se analizarán los nuevos planes y programas para el nivel medio superior en lo que concierne a la formación ciudadana.

\section{Programas vigentes de la SEP para la Educación Media Superior}

En México, la Educación Media Superior es parte de la educación obligatoria desde el año 2012, mediante modificación constitucional (DOF, 09/02/2012). Se estableció la obligación al Estado de ampliar cobertura de este nivel hasta alcanzar acceso universal para el grupo de población de 15 a 18 años, en el ciclo escolar 2021-2022. En este aspecto, de la oferta de servicios educativos, México ha abierto para sus generaciones jóvenes un derecho que en mucho rebasa el de otras naciones, ya que comprende desde el preescolar hasta el bachillerato; la mayoría de las naciones del planeta solo se han comprometido a brindar servicios educativos de primaria y secundaria. Dada la complejidad de nuestras sociedades, esto se considera un logro para la niñez mexicana.

El Sistema Nacional de Bachillerato (SNB) ha sido un esfuerzo de la Secretaría de Educación Pública por integrar a las escuelas que imparten educación escolarizada de nivel medio superior a un esquema de «evaluación», o quizá mejor de «auditoría». Con ello se busca que las instituciones cumplan con ciertos «estándares» mínimos de infraestructura, acervo bibliotecario, laboratorios equipados, talleres para la educación profesional, cumplimiento de currículos, 
perfiles docentes y prácticas cotidianas que permitan cumplir con la administración y la impartición de la educación, acorde a planes y programas establecidos para las diferentes modalidades que integran el Sistema. Se creó un consejo técnico independiente de la Secretaría de Educación Pública, encargado de «certificar» los planteles. Como se ha observado en varias instituciones, esta acreditación sirve para dignificar las escuelas y movilizar recursos desde la administración central de los subsistemas, con la finalidad de generar las condiciones mínimas en cuanto a equipamiento e infraestructura que permiten a los maestros desplegar un trabajo completo. La oferta se amplía y se dignifica. En cuanto a los aspectos académicos también se ha hecho un esfuerzo poco sistemático, pero ya prolongado por décadas, de acceder a enfoques que faciliten un mejor desarrollo de las nuevas generaciones.

A partir de 1970, el país ha experimentado muchos esfuerzos por reformar su sistema educativo, principalmente desde teorías cognitivas y socioculturales. Cada seis años surgen innovaciones en la educación, más en el discurso que en la práctica. Ya que el nivel medio superior no era obligatorio, seguía sus propias directrices, en las que cada subsistema respondía a otros sectores: el mundo laboral para las tecnológicas, al nivel superior en el caso de los bachilleratos generales y a diversas iniciativas en los casos de las escuelas privadas. A partir de 2012, este nivel entró a la misma lógica de las reformas impulsadas desde la autoridad central (federal, en México). Sin embargo, poco han permeado a las aulas en las que el tratamiento de todos los temas del currículo oficial se aborda desde un enfoque enciclopédico: los docentes se ocupan en adiestrar a los estudiantes para resolver exámenes como PLANEA y PISA; la enseñanza sigue al centro de la vida escolar; la fragmentación del conocimiento en asignaturas disciplinares y la desvinculación con respecto del contexto del entorno y de la población atendida, siguen prevaleciendo. Un número significativo de jóvenes adolescentes no comprenden para qué les sirven los conocimientos adquiridos y qué sentido asumirán en su vida. Si bien se habla de la autonomía curricular, abriendo espacios acotados por las «recomendaciones de la autoridad federal» para que los docentes realicen adaptaciones curriculares (SEP, 2018), en la práctica son pocos los maestros que se han atrevido a hacerlo, y, cuando lo hacen, son criticados ampliamente por sus pares, directivos y supervisores. 
Frente a este diagnóstico que, en términos generales, es compartido por la Secretaría de Educación en su página web, se plantea la última reforma (SEP, 2018). A partir del año 2012, la Federación ha trabajado en el establecimiento de un Marco Curricular Común (MCC) para todos los subsistemas del nivel medio superior, mismo que forma parte de la Ley General de Educación desde el año 2013 (DOF, 10 / 06 / 2013). La última innovación también plantea un MCC cuyos planes y programas de estudio fueron publicados en 2017 y se inició su implementación en el ciclo escolar 2018/2019. A continuación, se realiza un somero análisis de estos.

Como introducción a los planes, se explicitan las cuatro funciones del nivel medio superior: culminación de la educación obligatoria, formación ciudadana, preparación para ingresar al nivel superior y preparación para el trabajo (SEP, 2017, p. 12). El perfil de egreso presentado para los estudiantes, al finalizar el NMS, es muy ambicioso. Con las siguientes áreas se pretenden cubrir los elementos básicos de la cultura: la lengua materna, una lengua extranjera, el lenguaje matemático y el de las ciencias (tanto naturales, como sociales y humanas). Además, se establecen competencias en torno a la formación ciudadana que abarcan: incremento del pensamiento crítico, comprensión y participación en una democracia participativa e inclusiva, responsabilidades de mantenerse sano, contribuir a la sustentabilidad, apreciar las expresiones artísticas como elemento indispensable para la expresión humana y, por último, el adecuado desarrollo socio-emocional, que permite a los individuos un conocimiento de sí, a la vez que una apreciación y empatía por el otro y lo otro, expresada a través de relaciones sanas y constructivas. Por último, y como elemento nuevo en el discurso educativo oficial, deben impulsarse competencias que faciliten el perfeccionamiento de la imaginación y la creatividad (SEP, 2017, p. 23).

En otras palabras: un ciudadano bien provisto de las herramientas precisas para afrontar la vida en sociedades complejas del siglo XXI y para contribuir a los retos planteados por las problemáticas actuales. El discurso es congruente con los contenidos de la Convención Internacional de los Derechos del Niño y, con ello, el Estado Mexicano quiere aportar los elementos que le corresponden al pleno desarrollo de las nuevas generaciones. 
Sin embargo, si analizamos en términos técnicos las propuestas concretas de los programas, así como las sugerencias para su implementación, encontramos muchos problemas que se constituyen en obstáculos para alcanzar los propósitos enunciados. En las planeaciones se propone a los docentes explicitar el eje de trabajo, los componentes que lo conforman, los contenidos centrales, los contenidos específicos, los aprendizajes esperados y los productos esperados. Se describe cómo fomentar la interdisciplinariedad y cómo desarrollar diversas habilidades y competencias genéricas en todos los campos de estudio. Se introducen matrices de planeación que incluyen cómo las actividades abonan al perfil de egreso (SEP, 2017) y cómo se atienden los aprendizajes clave.

Se explicita que el campo de las Ciencias Sociales contribuirá prioritariamente a la formación ciudadana. Se piensa que la comprensión histórica y el análisis socioeconómico de nuestro país, así como la investigación social, resultarán en competencias ciudadanas. Si bien se transversalizan habilidades como la escucha, la argumentación, el análisis y el trabajo en equipo, entre otras, los ejercicios propuestos y la forma de resolver las adecuaciones curriculares dejan intactos los contenidos tradicionales. Así, por ejemplo, presenta el ejercicio de planeación sugerido con el desarrollo del tema de las guerras napoleónicas y su impacto en la Independencia de México (SEP, 2017, p. 420).

La planeación requerida a los maestros es cada vez más compleja y toma mucho tiempo su preparación, en lugar de simplificar la labor docente con propuestas prácticas del tratamiento de contenidos de interés general para los adolescentes. Los discursos en los programas de estudio están desgastados y no presentan la ruptura paradigmática necesaria para resolver las problemáticas planteadas en sus propios diagnósticos. La cantidad de contenidos y las horas de trabajo asignadas solo permiten un tratamiento superficial, teórico y simplificado de los temas a tratar. El desarrollo de la capacidad crítica requiere de tiempos reales para conocer el tema, de oportunidad para contrastar, de trabajo en equipo, de análisis, de búsqueda de argumentos y su fundamentación, de construcción de conocimientos en diferentes disciplinas, de reflexión individual y colectiva, de 
procesos metacognitivos para analizar cómo se está pensando y por qué. En doce o quince horas de trabajo es imposible realizar esto con temas tan complejos como, por ejemplo, «Perfiles demográficos y migraciones» (SEP. 2017, p. 430).

Los programas presentados siguen adoleciendo de los males que pretenden resolver: al principio plantean que serán menos contenidos para tratarse con mayor profundidad y que se vincularán a los contextos de la población adolescente. Los planes sí tocan temas de actualidad, pero son demasiados y no se les dota de un sólido aparato científico y crítico en su tratamiento, con la finalidad de abordar de una manera orgánica los conocimientos disciplinares, $y$, más importante aún, introducir a los jóvenes en las lógicas de la construcción del conocimiento en las diferentes disciplinas, desde la vinculación de estos temas con sus experiencias de vida personales y comunitarias.

Desde nuestra perspectiva, con un solo proyecto interdisciplinar sólido y profundo por semestre podría lograrse que los adolescentes realmente se adentraran en las implicaciones de cada disciplina, a la vez que pudieran abrirse los espacios para el aprendizaje enunciado en el propio plan de estudios (SEP, 2017, p. 27) y que, de trabajarse sistemáticamente, representaría una educación ciudadana real: propiciar el aprendizaje socioemocional, ético y cívico; incrementar relaciones constructivas en la diversidad, que incluyan la solución solidaria de conflictos emergentes; estar atentos a la equidad de género y a la inclusión de cualquier miembro del grupo; generar ambientes emocionalmente seguros en que los estudiantes pudieran conversar de temas y problemáticas que los aquejan, con la finalidad de abordarlos y trascenderlos; fomentar el cuidado de la persona, la empatía y las interacciones respetuosas entre los individuos; desarrollar la perseverancia y cuidar la honestidad, tanto en el trato entre personas, como en el ámbito académico.

El plan enfatiza estos aspectos que concretan una sólida educación ciudadana; sin embargo, no abre los espacios requeridos para acrecentarla a partir de un trabajo comprometido en torno a un proyecto con implicaciones personales y colectivas, preferentemente 
concretado a nivel comunitario. La formación ciudadana supone compromiso y actividades en las escuelas y comunidades: proyectos constructivos concretos (Gandhi, 2016).

\section{CONSTRUCCIÓN DE PAZ CON EQUIDAD Y ÉTICA: RETOS DE LA EMS}

¿Qué implicaciones tienen las actuales revoluciones tecnológicas en la Educación Media Superior? Si leemos posibles implicaciones en un escenario a cinco años, como expresan Demartini y Benussi (2017), o bien en prospectiva para el año 2030, en un primer momento debe pensarse en la pertinencia de los contenidos curriculares de la Educación Media Superior. En segundo lugar, es obligatorio el estudio interdisciplinario sobre cómo esas revoluciones están re-organizando el llamado mercado laboral, y sus alcances en la creación, adaptación y extinción de empleos (Mackinsey Global Institute, 2017 y 2018). En un tercer momento, se requiere analizar el estado de la Educación Media Superior, particularmente referido al enfoque en habilidades críticas, creativas, éticas, que posibiliten una cultura de bienestar (Tobin 2017), mismas que nutran la vida de un joven que se educa para la ciudadanía, libertad y equidad.

En un cuarto momento -y sin perder de vista el enfoque previo- es esencial revisar la relación entre espacios laborales, educación - es decir, diseño y actualización curricular- y el ser ser humano en el siglo XXI. Esta revisión es relevante, sobre todo, cuando se tiende a orientar la educación y sus contenidos curriculares hacia lo que la industria de la cuarta revolución requiere, como guía ubicua y preponderante: lo que se ha llamado The Next Production Revolution (OECD, 2017). Aunque es imprescindible la actualización de contenidos curriculares pensados para el entorno productivo actual, educar a niños y jóvenes es mucho más que orientarlos a considerar los entornos laborales que la industria necesita; esto es pertinente pero incompleto para guiar los propósitos fundamentales de la educación, en el sentido más amplio (Beck, Sollbrekke, Sutphen y Fremstad, 2015). Aclaramos: incompleto no significa erróneo. 
¿Qué otros aspectos han de considerarse para entender las implicaciones de estas revoluciones en la educación media superior? Estas implicaciones significan también prevenir los desajustes y las desigualdades que elevan la violencia, el desempleo, la injusticia y la ausencia de movilidad social. Enseguida nos referimos únicamente al esbozo de tres aspectos a considerar para prevenir y desarticular las implicaciones negativas de las actuales revoluciones tecnológicas:

a) Incorporar contenidos curriculares y extra curriculares que activen habilidades para construir una cultura y educación de paz, aplicada al entorno social, local, nacional y en conexión con el entorno mundial.

b) Incorporar contenidos curriculares, condiciones escolares y calidad de la enseñanza que construyan sostenidamente, y a largo plazo, la equidad en la educación atendiendo los entornos de desigualdad.

c) Incorporar contenidos curriculares y extra curriculares, prácticas de gestión educativa y formación docente guiados por la Ética Digital y la integridad académica, evidenciando su impacto en la vida laboral y la construcción de sociedades equitativas.

Estos tres aspectos están en riesgo de recibir menos atención cuando se actualizan o se rediseñan los contenidos curriculares, sobre todo en el contexto de las transformaciones tecnológicas recientes. Estos aspectos, entre otros que argumentaremos enseguida, tienen una relevancia humanística fundamental cuando los dimensionamos a largo plazo, es decir, para el presente y la configuración de un futuro que no debe ser el que se impone como determinante de la desigualdad. Sabemos que el futuro, en tanto tiempo que aún no existe, es una ilusión porque, al llegar al futuro esperado, solo hay presente: un instante cuya garantía es volverse pasado. Por ello, la propuesta sugerida radica en considerar los tres aspectos antes mencionados, como:

1. Criterios para orientar el rediseño o actualización curricular que permitan, a la Educación Media Superior, enfrentar los retos de los siguientes años para economías y sociedades en desventaja. 
2. Criterios de actualización de un modelo educativo «n».

3. Criterios para actualizar o construir lineamientos que nutran una educación para entornos creativos, adaptativos, interconectados, éticos, de integridad académica y resilientes.

4. Criterios que actualicen o re-estructuren la gestión educativa, administrativa y normativa para construir entornos que requiere el punto previo a este.

\section{No violencia para construir una cultura y educación de paz, aplicada al entorno social}

¿Por qué considerar la cultura y educación de paz como criterios para orientar la actualización tanto de los contenidos curriculares, entornos formativos, como de la gestión educativa, en el sistema de Educación Media Superior? Las estimaciones cuantitativas pueden proporcionarnos una idea panorámica de la importancia de la no-violencia, la cultura y educación de paz como criterios integrales de las dinámicas educativas del siglo XXI, especialmente en la atención educativa a niños y jóvenes. Un ejemplo de esas estimaciones está, entre otros estudios, en la cuantificación del costo de la violencia y el crimen en América Latina y el Caribe (Jaitman, 2017), que presenta el resultado del estudio en la región latinoamericana, en 2014: el 3.55\% del PIB de la región de ALC fue el costo de la violencia y el crimen, sin que esa cuantificación incluya los costos sociales, psicológicos, de salud, de desplazamiento o migración forzada, entre otros.

En el mismo estudio (Jaitman, 2017, pp. 31-32) leemos que estos costos superan a los del terrorismo o del cambio climático. Brasil, Centroamérica y México son los más afectados de la región de ALC (ver imagen 1). Si bien es un problema multifactorial, los costos están en aumento y los efectos son cada vez mayores como los observados en 2018, tanto en cifras del crimen como en los efectos de migraciones masivas centroamericanas por violencia regional. 


\section{Imagen 1. Costos por gobierno y costos por subregión de ALC}

Gráfico 2.3. Costos incurridos por el gobierno, por subregión, 2014 (como porcentaje del PIB)

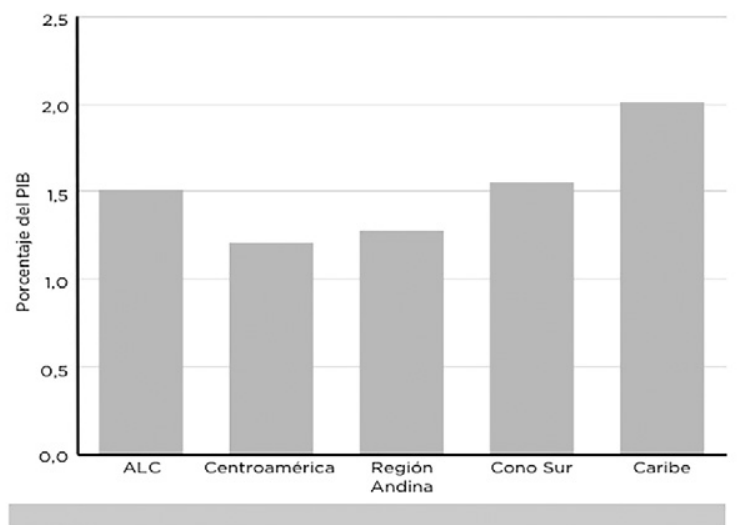

Fuente: Estimaciones propias en base a las fuentes citadas en el anexo de la Parte I.

Nota: ALC: América Latina y el Caribe.

Gráfico 2.4. Costos del crimen, por subregión, 2014 (como porcentaje del PIB)

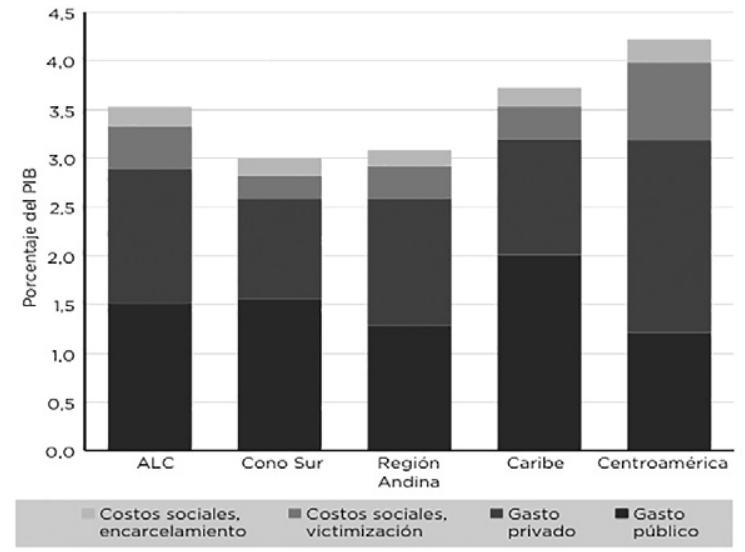

Fuente: Estimaciones propias en base a las fuentes citadas en el anexo de la Parte I.

Nota: ALC: América Latina y el Caribe.

Fuente: Jaitman Laura, ed. (2017, pp. 24 y 26). 
¿Cómo puede responder la educación media superior a este entorno de transformaciones? La violencia es un problema multifactorial, pero si reconfiguramos e intervenimos en uno de los factores donde reside el poder de transformación, la educación, puede pensarse de manera interrelacionada en cambios que fructifiquen la atención a las demás causas: equidad económica, de género, social, educativa, asimismo nutrir la Ética e integridad en la Educación Media Superior. La formación en cultura de paz y no violencia es plausible en los diseños curriculares y extracurriculares, es decir, como habilidades que se despiertan en la formación escolar y extra escolar, y al mismo tiempo, son puentes entre la escuela y la sociedad. Si examinamos con detenimiento los costos de la violencia y los dimensionamos a largo plazo, observamos un gasto que supera - y seguirá superando- la inversión en educación, salud y ciencia, entre otros.

La meta 16 de la Agenda 2030 para el Desarrollo Sostenible muestra que es prioritario: «promover sociedades pacíficas e inclusivas para el desarrollo sostenible [...]»(ONU, 2016, p. 19). La pregunta inmediata es ¿cómo lograrlo? Una ruta constructiva es la activación de la acción no violenta en entornos violentos, actitudes, habilidades, emociones, conocimiento, decisiones, resiliencia y atención consciente que permitan construir la disposición para la paz en niños y jóvenes. Si pensamos en la posibilidad de una ciudadanía global, ¿en qué declaraciones se fundamentan tanto la acción no violenta, como la cultura y educación de paz?, y ¿cómo deben considerarse para los diseños curriculares del sistema educativo de un país?

La resolución 55/282 (ONU, 7 de septiembre de 2001) de la Asamblea General, proclamó el Día Internacional de la Paz. El 15 de junio de 2007, con la resolución 61/271, la ONU declaró el 2 de octubre de cada año como «Día Internacional de la No Violencia» (ONU, 2007). En ambas resoluciones, como ejemplo en un conjunto de otras resoluciones y acciones de la ONU, observamos una evolución en la complejidad tanto de la idea de paz como de los debates, estudios e investigación en temas de paz (UNESCO, 2018, p. 30):

The definition of peace as the «absence of war» is probably the most popular and yet simplified definition of peace. However, numerous scholars and activists describe this narrow interpretation as «negative 
peace» across cultures. «Positive peace» has been posited as an alternative definition of peace embodying broader issues of development and social justice.

La base filosófica de la definición de paz puede variar, pero consideremos una visión educativa, social y aplicada de la idea de no violencia y cultura de paz, que tienen su horizonte en la idea de no violencia y paz positiva de Mahatma Gandhi: la no violencia activa y constructiva es ahimsa, en sánscrito. La paz es un derecho del ser humano, ocurre bajo las condiciones de un estado de justicia, equidad, bienestar para todo ser humano, es decir, incluyente. Sin embargo, la no violencia es una construcción activa, es una fuerza que radica en el ser humano y se fundamenta en el poder de la verdad: satya, en sánscrito. Gandhi (1960) concibe ahimsa como fuerza activa que coexiste con la justicia, fuerza constructiva que reposa en satya: la verdad se realiza de manera activa.

En suma, la cultura y educación de paz, como un eje formativo en el entramado de la ciudadanía global, se entiende como construcción y no significa una asignatura cuantificable, que se vuelva un fin medido por un examen con valor numérico. En la idea y práctica de «construcción» está la inquietud de cómo lograrlo, la propuesta reside en una visión estética, creativa, poética de la no violencia. La acción constructiva es creación y renovación. También es libertad en tanto es un fin en sí misma; la disposición diaria que se despierta y permite responder de manera no violenta: esa es precisamente la disposición que se despierta en la educación para la paz. Esa construcción diaria es creativa, y por ello, mencionamos antes, es poiesis (Martínez Ruiz, 2016 y 2018): es poética educativa, es un camino estético y no acumulación de conceptos e información. Distinguimos esta vía como una estética de la no violencia, marga, odos, camino, un método para la construcción de paz, cuya base es la libertad.

\section{Equidad en la educación}

La idea de equidad en la educación ha sido una construcción retórica para muchos discursos; la importancia que queremos subrayar va 
más allá de eso. Nuestro tiempo es un tiempo de resignificación: ¿qué entendemos por equidad educativa? De manera general, la equidad educativa no se refiere a homogeneizar la expectativa de obtener los mismos resultados de desempeño escolar, es decir: que los estudiantes de un país - en este caso, México- obtengan los mismos resultados al ser medidos, «[...] sino que las diferencias en los resultados de los estudiantes, como el rendimiento académico, los resultados sociales y emocionales, y el nivel de escolaridad que alcancen, no dependen de sus antecedentes socioeconómicos [...]»(OECD, 2018). La equidad educativa se entiende como el mecanismo que posibilita el mismo horizonte de oportunidad para todos los estudiantes de un sistema educativo, sin importar su procedencia socioeconómica.

¿Por qué la equidad está indisolublemente relacionada con la construcción y educación para la paz? Esta vinculación reside en que, entre las diversas causas que catalizan la violencia, está la desigualdad, la falta de oportunidades, la ausencia de condiciones para lograr una vida digna con un ingreso equitativo. Ambas - cultura de paz y equidad educativa - constituyen la idea de ciudadanía global, es decir, una ciudadanía con equidad; las dos son condiciones que posibilitan la movilidad social. Martha Nussbaum identificó una capacidad básica de lo que llama ciudadanía mundial: la «imaginación narrativa» (Nussbaum, 2005, p. 30): «Esto significa la capacidad de pensar cómo sería estar en el lugar de otra persona, y comprender las emociones, los deseos y anhelos que alguien así pudiera experimentar».

La «imaginación narrativa» es un ejercicio filosófico de aprendizaje que ayuda a entender el lugar e importancia de la equidad, el sufrimiento ajeno y la conciencia de que no todos parten de un mismo piso nivelado y equitativo, sino desigual, y que no debe de ser así; todo ello es un ejercicio de ciudadanía. Nuestro tiempo exige una traducción de ese contexto y por ello se requiere evitar que: «[...] las naciones de todo el mundo en breve producirán generaciones enteras de máquinas utilitarias, en lugar de ciudadanos cabales con la capacidad de pensar por sí mismos [...]» (Nussbaum, 2010, p. 20). Esta misma conciencia de equidad en nuestro siglo se asocia a otra conciencia que nos constituye: el ethos, cuya relevancia cobra una dimensión crucial en este siglo. Concretamente en el horizonte educativo para jóvenes, aparece la necesidad de una conciencia práctica, nutrida, practicada, experimentada en 
situaciones cotidianas en el entorno educativo $y$, posteriormente, como fundamento de la vida laboral y la construcción de sociedades equitativas: me refiero a la Ética Digital y a la integridad académica. La breve reflexión queda esbozada enseguida.

\section{Ética Digital e integridad académico-laboral}

¿Qué implicaciones éticas, legales y laborales han tenido las innovaciones tecnológicas, el desarrollo de tecnología inteligente y la conectividad global para la Educación Media Superior? Un marco de referencia para contextualizar estas implicaciones es el Manifiesto Onlife (2015) que desarrolla un marco filosófico, ético y legal de la interacción entre el ser humano, la máquina y la naturaleza. Otra iniciativa relacionada para identificar problemáticas éticas que impactaron la educación, la mediación comunicativa y las relaciones humanas es ONLIFE Initiative: Concept Reengineering for Rethinking Societal Concerns in the Digital Transition (European Commission, 2013). En el marco de esta iniciativa, esbozamos algunas implicaciones a considerar: «[...] el cambio de enfoque que va de la prioridad en las entidades a la prioridad en las interacciones [...]» $(2015$, p. 7$)$. Cada una de las transformaciones detonadas por las revoluciones tecnológicas requiere una resignificación y traducción a los nuevos contextos, de la idea misma de educación y sus relaciones con el empleo y la sociedad.

La idea de Ética Digital es una respuesta al entorno educativo que incorporó - e incrementará- el empleo de tecnología en sus ambientes de aprendizaje (e-learning, b-learning, m-learning) y la expectativa de crecimiento en América Latina. Sin embargo, en este entorno, ¿cómo define un joven de nuestro tiempo su horizonte de valores? ¿Qué ha sido transformado por la revolución tecnológica? Si somos consecuentes con la idea de revoluciones tecnológicas actuales, entonces parece inevitable pensar el desplazamiento de ideas éticas y posicionamiento de valores. En Filosofía, como lo explica Floridi (2014), las tres revoluciones previas han implicado un cambio de paradigmas y referentes cognitivos para entender el universo y al ser humano: la revolución copernicana que reconsideró dónde estamos y la relación sujeto-objeto; seguida de la segunda revolución, con Darwin y su investigación sobre 
nuestro lugar en la evolución, y la tercera configurada por Freud y el entendimiento de aquello que nos gobierna y que actúa en el interior del sujeto. Para Floridi, la cuarta revolución radica en lo iniciado por Alan Turing y que constituyó otra revolución cognitiva que reconfigura el entendimiento sobre nosotros y el entorno.

Por otra parte, desde la perspectiva de transformación tecnológica y de producción, las revoluciones que nos preceden se entienden como una sucesión enlazada de transformaciones tecnológicas (Xing y Marwala, 2017; Demartini y Benussi, 2017). La primera revolución fue catalizada por los descubrimientos de Newton y la posibilidad de construir motores de vapor para una industria que desplazó mano de obra. La segunda residió en las transformaciones en la producción y en la vida que trajo la electricidad. La tercera es entendida como la revolución electrónica y los desarrollos que dependían del uso de descubrimientos, como el transistor (Xing, Marwala, 2017, p. 11). La digitalización, la inteligencia artificial, el desarrollo en machine learning, la automatización de la industria, la integración horizontal y vertical de los procesos de producción, entre otros elementos, definen la llamada «cuarta revolución industrial».

En medio de las revoluciones tecnológicas actuales, cuestionar es un ejercicio imprescindible: ¿cómo desentrañar el sentido histórico de nuestro tiempo, sin perder de vista el propósito más elevado de la educación que recibirán niños y jóvenes en los siguientes años? En este contexto, los diseños, rediseños y las actualizaciones curriculares para la Educación Media Superior presentan desafíos que no son exactamente tecnológicos, sino que ha de considerarse la capacidad creativa, reflexiva y práctica para entender y emplear la tecnología actual, como un instrumento que permita condiciones para la paz, la equidad y para nutrir entornos donde la Ética e integridad sean las guías de acción. Allí es precisamente donde un Marco Curricular Común (MCC) abre un horizonte para los demás contenidos curriculares y puede interrelacionarlos. Los retos de la actual Educación Media Superior en México no son diferentes de los desafíos globales; por la manera en que comprendamos esos retos y percibamos su naturaleza humana y ética, podremos concebir mejor el papel de la educación en las actuales revoluciones tecnológicas. Asumirlas desde el aspecto industrial, y de ahí 
buscar que la educación se adapte a las necesidades productivas, es un empobrecimiento de las posibilidades humanas.

\section{CONCLUSIONES}

En el actual sistema de Educación Media Superior mexicana, el MCC se sigue presentando con una serie de asignaturas que contienen muchos contenidos y pocas herramientas para el perfeccionamiento de las competencias ciudadanas que se pretenden alcanzar. Los docentes, egresados casi todos de una licenciatura disciplinar, en su mayoría no poseen las herramientas académicas ni de desarrollo personal para abordar con imaginación y creatividad una verdadera reforma educativa. Entre los retos más importantes está ciertamente el impulso de todas las capacidades académicas y de perfeccionamiento humano requeridas para el ejercicio ciudadano en sociedades complejas que, en sí mismas, poseen un enorme dinamismo difícilmente aprehensible desde posturas críticas y comprometidas.

La construcción de paz con equidad y ética, constituye uno de los retos de la Educación Media Superior en este tiempo, particularmente en sistemas educativos con las condiciones socioeconómicas y demográficas mexicanas, considerando las expectativas poblacionales de Latinoamérica y México para los próximos años (US Census Bureau, 2018). Estas condiciones también pueden pensarse en el contexto de las implicaciones de las actuales revoluciones tecnológicas — para cada tipo de bachillerato y sus condiciones regionales-, donde observamos la visión crítica a largo plazo sobre una doble consideración: ¿formamos a los jóvenes que cursan la EMS para los entornos actuales, y los ambientes académicos y laborales de los próximos años? Y por otra parte, la revisión, actualización y/o rediseño de un MCC de la EMS, en México, no puede dejar de lado las implicaciones éticas, legales y laborales que han sido catalizadas por las innovaciones tecnológicas y el desarrollo de tecnología inteligente. 


\section{REFERENCIAS}

Ambramovich, V. (2006) «Una aproximación al enfoque de derechos en las estrategias y políticas de desarrollo». Revista CEPAL, vol. 88, pp. 35-50. Consultado: octubre 12, 2018. Recuperado de https://repositorio.cepal.org/handle/11362/11102

Ariès, P. (1987). El niño y la vida familiar en el Antiguo Régimen. España. Ed. Taurus.

Aristóteles (2000). Política. Versión de Antonio Gómez Robredo. Col. Bibliotheca Scriporum graecorum et romanorum mexicana. México: UNAM.

Beck, E., Sollbrekke, T., Sutphen, M. y Fremstad, E. (2015). «When mere knowledge is not enough: the potential of bildung as self-determination, co-determination and solidarity». Higher Education Research E Development, vol. 34, n. 3, pp. 445-457.

Demartini, C. y Benussi, L. (2017). «Do Web 4.0 and Industry 4.0 Imply Education X.0?». IT Pro May/June 2017. IEEE Computer Society. Recuperado de: https: / / pdfs.semanticscholar.org/e0b9/45ce38d3ea638de3c02f069a846cde9651fe.pdf

De Sousa Santos, B. (1997) «Uma concepçao multicultural de direitos humanos». Revista lua nova, n. 39, pp. 105-124. Issn 0102-6445. Consultado: octubre 8, 2018. Recuperado de http:/ / dx.doi.org/10.1590/ S0102-64451997000100007

DOF (2012) Diario Oficial de la Federación, 09/02/2012, DECRETO por el que se declara reformado el párrafo primero; el inciso c) de la fracción II y la fracción $V$ del artículo 3o., y la fracción I del artículo 31 de la Constitución Política de los Estados Unidos Mexicanos. Consultado: junio 24, 2012. Recuperado de http:/ / www.dof.gob.mx/ nota_detalle.php?codigo $=5233070 \&$ fecha $=09 / 02 / 2012$ 
DOF (2013). Diario Oficial de la Federación. 10/06/2013. DECRETO por el que se reforman los artículos 3o., 4o., 90., 37, 65 y 66; y se adicionan los artículos 12 y 13 de la Ley General de Educación. Consultado: octubre 10, 2017. Recuperado de http:// www.dof.gob.mx/nota_detalle. php\%3Fcodigo\%3D5301832\%26fecha\%3D10/06/201

European Commission (2013). ONLIFE Initiative: Concept Reengineering for Rethining Societal Concerns in the Digital Transition. Bruselas 8 de septiembre. Consultado: octubre 22, 2018. Recuperado de https: / / ec.europa.eu/digital-single-market/en/news/onlife-initiativeBruselas

Floridi L. (ed.), (2015). The Onlife Manifesto. Being Human in a Hyperconnected Era. Springer. DOI 10.1007/978-3-319-04093-6

Gandhi, M.K. (1960). All men are brothers. Ahmedabad, India: Navajivan Trust.

Gandhi, M.K. (2016). El Programa Constructivo. Su significado y lugar. Biblioteca Gandhi, Ciudad de México: El Colegio de San Luis, Gujarat Vidyapith, OraWorldMandala, Instituto Politécnico Nacional y La Gandhiana.

García Méndez, E. (1998). Infancia de los Derechos y la Justicia. Consultado: Consultado febrero 18, 2007. Recuperado de http:/ / docvirt. com / docreader.net / DocReader.aspx?bib=bibliotdca\&pagfis $=4252$

Jaitman, L. (Ed.). (2017) Los costos del crimen y de la violencia: nueva evidencia y hallazgos en América Latina y el Caribe. Monografía del BID: New York. Recuperado de http:/ / dx.doi.org/10.18235/0000615

Kincheloe J.L. y S.R.Steinberg. (2012). Repensar el multiculturalismo. Traducción de J. Real. Col. Repensar la educación, n. 8. Barcelona: Ediciones Octaedro.

Marre, D. (2014) «Prólogo» en Llovet, V. Pensar la infancia desde América Latina. Un estado de la cuestión. Col. Red de Posgrados en Ciencias Sociales. Buenos Aires: Clacso (pp. 9-26).

Martínez Ruiz, X. (2016). Poética educativa. Artes, educación para la paz y atención consciente. Colección Paideia Siglo XXI, México: IPN. Recuperado de: http:/ / www.innovacion-omp.ipn.mx/index.php/ practica_educativa/catalog/view/5/3/224-1 
Martínez Ruiz, X. (2017) «Onlife Manifesto y la ética digital». Revista mexicana de bachillerato a distancia, n. 18. Agosto. México: UNAM.

Martínez Ruiz, X. (2018). «The alignment argument: at the crossroads between mindfulness and metacognition». Learning: Research and Practice, 4:1, 29-38, DOI: 10.1080/23735082.2018.1428096. Recuperado de: https: / / www.tandfonline.com/ doi / abs / 10.1080 / 23735082.2018. 1428096

Mackinsey Global Institute (2017). Jobs lost, Jobs Gained: Workforce transitions in a time of automation. Recuperado de: www.mckinsey. $\mathrm{com} / \mathrm{mgi}$

Morin, E. (1999). Los siete saberes necesarios para la educación del futuro. Traducción de Mercedes Vallejo- Gómez. Paris: UNESCO.

Nussbaum, M.C. (2005). El cultivo de la humanidad. Una defensa clásica de la reforma en la educación liberal. Barcelona: Ediciones Paidós Ibérica.

Nussbaum, M.C. (2010). Sin fines de lucro. Por qué la democracia necesita de las humanidades. Buenos Aires: Katz Editores.

OECD (2017). The Next Production Revolution: Implications for Governments and Business, OECD Publishing, Paris.

Organización de las Naciones Unidas (ONU) (15 de enero de 1998a). Proclamación del año 2000. Año Internacional de la Cultura de Paz, Resolución de la Asamblea General de las Naciones Unidas, n. A/ RES/52/15. Nueva York, EE UU: ONU. Recuperado de: https: / / undocs.org/es/A/RES/52/15

Organización de las Naciones Unidas (ONU) (2001). Proclamación del Día Internacional de la Paz, Resolución de la Asamblea General de las Naciones Unidas, n. A/RES/55/282. Nueva York, EE UU: ONU. Recuperado de: https:/ / undocs.org/es / A/RES/55/282

Organización de las Naciones Unidas (ONU) (2007). Proclamación del Día Internacional de la No Violencia, Resolución de la Asamblea General de las Naciones Unidas, n. A/RES/61/271. Nueva York, EE UU: ONU. Recuperado de: http:/ / www.un.org/es/events/nonviolenceday / 2007/ resolution.shtml 
Pardo, P. (2018). «Los adolescentes se alzan contra las armas en EEUU». Periódico El Mundo, Sección Internacional. España. Consultado: febrero 22, 2018. Recuperado de https:/ / www.elmundo.es/internacional $/ 2018 / 02 / 22 / 5 a 8 d$ cf57268e3ec8518b45d7.html.

Pries, L. (2005). "Configurations of Geographic and Societal Spaces: a Sociological Proposal between "Methodological Nationalism" and the "Spaces of Flow"». Global Networks, a Journal of Transnational Affairs, vol. 5, n. 2. Abril. Pp. 167-190. Consultado: enero 28, 2006. Recuperado de https://onlinelibrary.wiley.com/doi/pdf/10.1111/ j.1471-0374.2005.00113.x

Sen, A. (1982). Choice, Welfare and Measurement. Oxford: Basil Blackwell.

SEP (2017). Planes de estudios de referencia del componente básico del marco curricular común de la educación del nivel medio superior. México. SEP. Consultado: agosto 10, 2018. Recuperado de https:// www.gob.mx/ cms/uploads/attachment/file/241519/planes-estudio-sems.pdf

SEP (2018). ¿Por qué actualizar el currículo de la Educación Media Superior? Video consultado: septiembre 30, 2018. Recuperado de htpps:// www.gob.mx/nuevomodeloeducativo

Tobin, K. (2017). «Researching mindfulness and wellness». In M. Powietrzynska, \& K. Tobin (Eds). Weaving complementary knowledge systems and mindfulness to educate a literate citizenry for sustainable and healthy lives (pp. 1-19). Rotterdam, The Netherlands: Sense Publishing.

Xing, Bo, \& Marwala T. (2017). «Implications of the Fourth Industrial Age for Higher Education». The Thinker, vol. 73. Quarter 3. Recuperado de: http: / / www.thethinker.co.za/resources/Thinker\%2073.pdf 